\title{
An Iterative based Feedforward-Feedback Control Approach to High-Speed AFM Imaging
}

\author{
Ying $\mathrm{Wu}^{\dagger} \quad$ Qingze $\mathrm{Zou}^{\ddagger}$ \\ Mechanical Engineering Department, Iowa State University, Ames, IA 50011
}

\begin{abstract}
AFM imaging requires precision positioning of the AFM probe relative to the sample in all $x-y-z$ axes, especially the vertical $z$-axis direction. Recently, the currentcycle-feedback iterative-learning-control (CCF-ILC) approach is proposed for high-speed AFM imaging. The CCF-ILC feedforward-feedback 2 degree-of-freedom (DOF) controller design has been successfully implemented for iteratively imaging on one scanline. In this article, we extend this CCFILC approach to the entire imaging of samples. The main contribution of this article is the analysis and the use of the CCF-ILC approach for tracking sample profiles with variations between scanlines (called line-to-line sample variations). The convergence (stability) of the CCF-ILC system is analyzed for the general case where the line-to-line sample variation occurs at each iteration. The allowable line-to-line sample profile variation is quantified. The performance improvement of the CCF-ILC is discussed by comparing the tracking error of the CCF-ILC technique to that of using feedback control alone. The proposed CCF-ILC control approach is illustrated by implementing it to the $z$-axis direction control in AFM imaging. Experimental results show that the imaging speed can be significantly increased by using the proposed approach.
\end{abstract}

\section{INTRODUCTION}

In this article, we extend recently developed CCF-ILC approach from high-speed AFM imaging of one scanline to the entire imaging of the samples. Since its invention [1], AFM has been established as an enabling tool to image as well as to manipulate matters at nanoscale [2]. Current AFM system, however, is slow and AFM imaging, therefore, is time consuming. Although precision positioning of the AFM-probe in high-speed lateral $x-y$ axes scanning can be achieved by using recently-developed control techniques [3], [4] such as the inversion-based iterative learning control [3], challenges exist in the precision positioning of the AFM-probe in the vertical $z$-axis.

Efforts have been made to improve the $z$-axis precision positioning of the probe during AFM imaging. For example, Robust feedback control [4] and 2DOF control system [5] have been used in AFM systems to increase the AFM imaging bandwidth. However, the bandwidth and the robustness of feedback-based approaches are limited by the fundamental constraints of closed-loop feedback control. And in previous 2DOF controller design, feedforward controller is designed to be causual and the current feedforward control signal was generated by using the total control signal from the last scanline in [5], which limit the

† E-mail:wuying@iastate.edu.

$\ddagger$ Corresponding author, E-mail: qzzou@iastate.edu. performence of previous 2DOF control approach.

Recently, the current-cycle-feedback iterative-learningcontrol (CCF-ILC) approach is proposed in [6] for highspeed AFM imaging. The CCF-ILC approach integrated the $H_{\infty}$ robust control technique [7] with the inversion-based feedforward control technique [8], [9]. Particularly, the feedback controller is designed based on the $H_{\infty}$ robust control theory, and the feedforward controller is designed based on the recently-developed robust-inversion technique [10] to overcome the causality limits and explicitly account for the uncertainties of system dynamics when finding the inverse feedforward controller [10]. Moreover, iteration is introduced to further exploit the priori knowledge of the sample profile in the AFM imaging, as well as to compensate for the disturbances and uncertainties effects. As the first step, the CCF-ILC 2DOF controller design has been successfully implemented in [6] to iteratively image on the same scanline. In this article, we extend this CCF-ILC approach to the entire sample imaging.

The main contribution of this article is the analysis and the use of the CCF-ILC approach for AFM $z$-axis positioning with line-to-line sample variations. The proposed CCF-ILC control approach is illustrated by implementing it to the $z$-axis direction control in AFM imaging. Experimental results show that the speed of obtaining an entire image can be significantly increased by using the proposed approach.

\section{Problem Formulation And Convergence ANALYSIS}

In this section, we formulate and discuss the CCF-ILC approach to the vertical positioning of the AFM probe, when there exists line-to-line sample variation. We start with briefly describing the feedback control system of $z$-axis for the AFM imaging.

In general, the feedback control system for $z$-axis AFM probe positioning can be schematically presented by Fig. 1(a), where $G_{z}(s)$ denotes the dynamics model of the piezo actuator for the $z$-axis positioning, $G_{c}(s)$ denotes the cantilever dynamics (from the output of the $z$-axis piezo to the cantilever deflection), $K_{s}(s)$ denotes the photodiode sensitivity, $d_{s}(\cdot)$ denotes the sample profile, and $n_{s}(\cdot)$ denotes the system noise. The goal of the $z$-axis AFM probe positioning is to maintain a constant setpoint value (i.e., constant normal force between the tip and the sample) during the scanning process. Then the image of the sample topography can be estimated using the control signal or the deflection error [6]. 

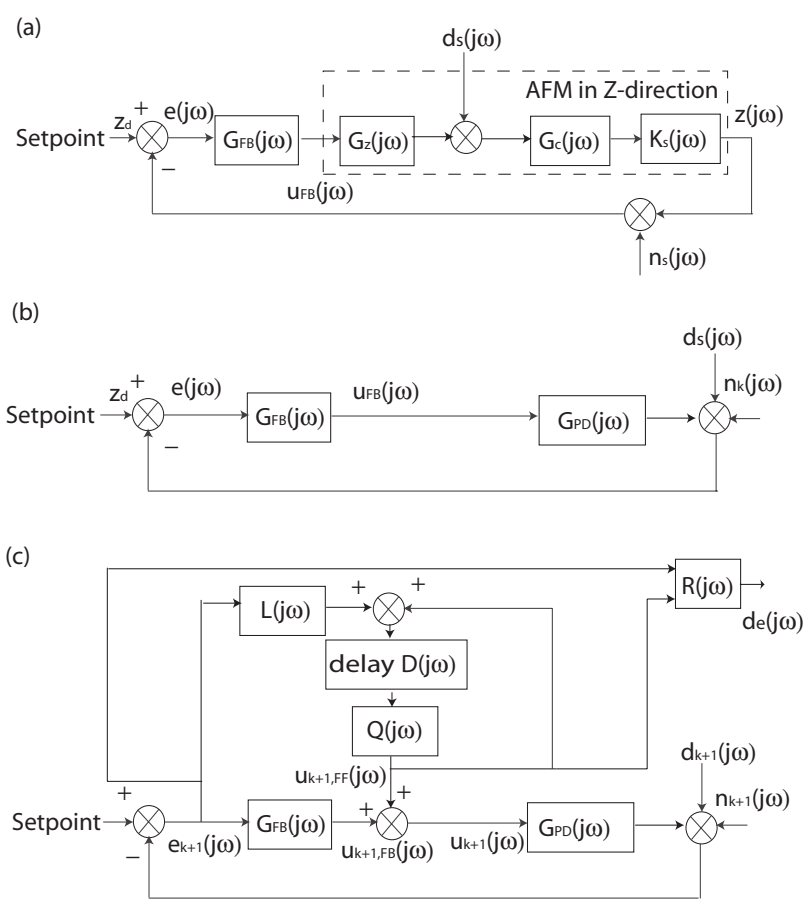

Fig. 1. The block diagram of (a) a standard feedback loop, (b) the modified feedback loop, and (c) the proposed CCF-ILC approach for the vertical $z$ axis positioning in AFM imaging.

Unlike the above feedback-based approach to $z$-axis positioning, the proposed CCF-ILC approach integrates an online iterative-learning control (ILC) as feedforward to the $z$ axis feedback control. First, to simplify the presentation of the controller design, the unknown sample profile is scaled with the DC-gain of the cantilever-photodiode dynamics $G_{c}(0) K(0)$, and then right-shifted to the joint point at the cantilever deflection output in the control block diagram, as shown in Fig. 1 (b) (The same notation $d_{s}(\cdot)$ is used for simplification). Thus, the proposed CCF-ILC controller can be schematically shown in Fig. 1 (c), where $Q(j \omega)$ and $L(j \omega)$ are the ILC filters to be designed, 'delay $D(j \omega)$ ' denotes the one-scanline-period delay, and $R(j \omega)$ denotes the observer to be designed for obtaining the measured sample profile $d_{e}(j \omega)$. Specifically, the objectives of the proposed CCF-ILC design are that, during the entire imaging process, the CCF-ILC system should

1) guarantee the convergence of the CCF-ILC approach, i.e., the feedforward control input $u_{k, F F}(j \omega)$ remains bounded for all iterations $(\forall k>1)$ when there exists line-to-line sample variations between each iteration, and the residual error $e_{k}(j \omega)$ converges to zero when the noise $n(j \omega)$ and the line-to-line sample variation vanishes;

2) improve the output tracking with the augmented feedforward control, i.e., for the same feedback controller, the tracking error $e(j \omega)$ (e.g., the deflection signal) when using the CCF-ILC approach is smaller than that when using the feedback control alone; and

$3)$ improve the imaging accuracy (denoted as $d_{e}(j \omega)$ in Fig. 1 (c)), i.e., the estimation of the sample profile $d_{s}(j \omega)$, is more accurate than the estimation obtained by using current commercial AFMs.

In the proposed CCF-ILC technique, a stabilizing feedback controller $G_{F B}(s)$ is designed first-the feedback controller $G_{F B}(s)$ guarantees that the feedback loop is internally stable [7]. This implies that the first objective of the proposed controller design requires all the signals in the control system (Fig. 1 (c)) to be bounded throughout the iterations. It is noted that the $z$-axis positioning of the AFM probe can be sensitive to effects such as the variation of the cantilever type and/or mounting, the setpoint value of the loading force (i.e., the cantilever deflection), and the measurement noise. Therefore, the feedback controller is designed to enhance the robustness of the entire control system against these adverse effects. We design the feedback controller by using the robust control technique [6], [10] (The readers are referred to [10] for details). In the following, we assume that such a feedback controller has already been in place.

\section{A. CCF-ILC Design: Convergence Analysis}

In the proposed CCF-ILC approach, the following general form of the iterative learning control law is employed :

$$
\begin{aligned}
u_{0, F F}(j \omega) & =0 \\
u_{k+1, F F}(j \omega) & =Q(j \omega)\left(u_{k, F F}(j \omega)+\right. \\
& \left.L(j \omega) e_{k}(j \omega)\right), \\
& \text { for } k \geq 1
\end{aligned}
$$

where $Q(j \omega)$ and $L(j \omega)$ are the ILC filters to be designed, and the filter $Q(j \omega)$ is factored out without loss of generality. We note that the CCF-ILC approach can be implemented in several different schemes. For example, the CCF-ILC algorithm can be applied to repeatedly image on the first scanline until the required imaging precision (i.e., tracking precision) is achieved before moving to image at the next scanline. Or alternatively, the CCF-ILC approach can be applied to image at each scanline with a pre-chosen number of iterations. These schemes are all special cases of the more general one where the line-to-line sample profile variation occurs at each iteration. Thus, we discuss next the conditions to guarantee the convergence of the CCF-ILC law (the first design objective) for this general scenario.

Lemma 1: Let $G_{P D}(j \omega)$ be the frequency response of a linear time invariant plant, $G_{F B}(j \omega)$ be a stabilizing feedback controller, and let $S(j \omega)$ be the closed-loop sensitivity transfer function. Then for bounded measurement noise $n_{k}(j w)$ during the $k^{t h}$ iteration, i.e., $\left|n_{k}(j \omega)\right| \leq \delta(\omega)$, and bounded modified line-to-line sample variation as defined below,

$$
\begin{aligned}
\widehat{\Delta d}_{k}(\omega) & \triangleq\left|Q(j \omega) d_{k}(j \omega)-d_{k+1}(j \omega)\right| \\
& \leq \max _{k} \widehat{\Delta d}_{k}(\omega) \triangleq \widehat{\Delta d}_{\max }(\omega)
\end{aligned}
$$

both the iterative control input $u_{k}(j \omega)$ and the residual error $e_{k}(j \omega)$ are bounded throughout the iterations, provided that the following iteration coefficient $\rho(j \omega)$ is less than one, i.e.,

$$
\rho(\omega)=\left|Q(j \omega)\left\{1-G_{P D}(j \omega) S(j \omega) L(j \omega)\right\}\right|<1 .
$$


Moreover, the residual error $e_{k}(j \omega)$ is bounded by an affine function of the maximum modified line-to-line sample variation $\widehat{\Delta d}_{\max }(j \omega)$ and the noise level $\delta(\omega)$

$$
\begin{aligned}
\left|e_{\infty}(j \omega)\right| & \triangleq \lim _{k \rightarrow \infty}\left|e_{k}(j \omega)\right| \\
& \leq\left|\frac{S(j \omega)}{1-\rho(\omega)}\right|\left|\widehat{\Delta d}_{\max }(j \omega)\right|+\mathbf{E}_{\delta}(j \omega) \delta(\omega),
\end{aligned}
$$

where the limit of the residual error $e_{\infty}(j \omega)$ is called the ultimate ILC error, and the frequency dependent coefficient $\mathbf{E}_{\delta}(j \omega)$ is defined as

$$
\mathbf{E}_{\delta}(\omega) \triangleq \frac{\left|Q(j \omega) G_{P D}(j \omega) S(j \omega) L(j \omega) S(j \omega)\right|+|S(j \omega)|}{|1-\rho(\omega)|}
$$

Proof We proceed by quantifying the residual error $e_{k}(j \omega)$ for given noise/disturbance $n_{k}(j \omega)$. Note that from the block diagram in Fig. 1 (c), for any given stabilizing feedback controller, the feedforward control input $u_{k, F F}(j \omega)$ in the $k^{t h}$ iteration can be written as

$$
\begin{aligned}
& u_{k, F F}(j \omega)=S^{-1}(j \omega) G_{P D}^{-1}(j \omega) \\
& \quad\left[-e_{k}(j \omega)-S(j \omega) d_{k}(j \omega)-S(j \omega) n_{k}(j \omega)\right]
\end{aligned}
$$

In the following derivation, the dependence on $j \omega$ is omitted for economy. Substituting Eq. (6) into Eq. (1) leads to

$G_{P D}^{-1}\left[-e_{k+1}-S d_{k+1}-S n_{k+1}\right]=Q G_{P D}^{-1}\left[-e_{k}-S d_{k}-S n_{k}\right]$ $+S Q L e_{k}$

Then the residual error at the next iteration $k+1$ can be obtained from Eq. (7) as

$$
\begin{aligned}
e_{k+1}= & Q\left(1-G_{P D} S L\right) e_{k}+\left(Q d_{k}-d_{k+1}\right) S \\
& +Q S n_{k}-S n_{k+1} \\
= & \left\{Q\left(1-G_{P D} S L\right)\right\}^{k+1} e_{0} \\
& +\sum_{j=0}^{k}\left\{Q\left(1-G_{P D} S L\right)\right\}^{j}\left(Q d_{k}-d_{k+1}\right) S \\
& +\sum_{j=0}^{k}\left\{Q\left(1-G_{P D} S L\right)\right\}^{j} Q S n_{k-j} \\
& -\sum_{j=0}^{k}\left\{Q\left(1-G_{P D} S L\right)\right\}^{j} S n_{k+1-j}
\end{aligned}
$$

By changing the index used in the last term in Eq. (8), the last two terms in Eq. (8) can be simplified as:

$$
\begin{aligned}
& \left\{\sum_{j=0}^{k}\left\{Q\left(1-G_{P D} S L\right)\right\}^{j} Q G_{P D} S L S n_{k-j}\right\} \\
& -s n_{k+1}+\left\{Q\left(1-G_{P D} S L\right)\right\}^{k+1} s n_{0}
\end{aligned}
$$

Substituting Eq. (9) into Eq. (8) and using triangle inequality, the iterative residual error $e_{k+1}$ can be bounded as

$$
\begin{aligned}
\left|e_{k+1}\right| \leq & \left|\left\{Q\left(1-G_{P D} S L\right)\right\}\right|^{k+1}\left|e_{0}\right| \\
& +\sum_{j=0}^{k}\left|\left\{Q\left(1-G_{P D} S L\right)\right\}\right|^{j}\left|\left(Q d_{k}-d_{k+1}\right) S\right| \\
& +\sum_{j=0}^{k}\left|Q\left(1-G_{P D} S L\right)\right|^{j}\left|Q G_{P D} S L S\right|\left|n_{k-j}\right| \\
& +\left|\left\{Q\left(1-G_{P D} S L\right)\right\}^{k+1} S n_{0}-S n_{k+1}\right| \\
\leq & \rho^{k}\left|e_{0}\right|+\frac{\widehat{\Delta d_{\max } S\left(1-\rho^{k}\right)}}{1-\rho} \\
& +\frac{\left|Q G_{P D} S L S\right||\delta|\left(1-\rho^{k}\right)}{1-\rho} \\
& +\left(1+\rho^{k+1}\right)|S||\delta| .
\end{aligned}
$$

Thus, it is evident from the above Eq. (10) that when the iteration coefficient $\rho(\omega)<1$, the tracking error $e_{k}$ is bounded for all iterations $k \geq 1$, and the ultimate error, $\left|e_{\infty}\right|$ is eventually bounded as in Eq. (4) when $k \rightarrow \infty$. Similarly, it can be shown that under the same condition (i.e., $|\rho(\omega)|<1$ ), the iterative control input, $u_{k}(j \omega)$, is also bounded for all iteration $k \geq 1$. This completes the proof. It is noted that, for the special case of Lemma 1 where the line-to-line sample variation vanishes as in [6], the CCF-ILC law (1) is also converge.

Lemma 1 implies that when the noise effect is small $(\delta(j \omega) \rightarrow 0)$, the bound of the residual error is monotonically decreasing with iterations (see Eq. (10)). Thus, to guarantee the overall imaging quality in implementations, it is preferred to iteratively image on one scanline until the residual error (7) is smaller than a user-chosen thresh-hold value, then proceed the imaging on the rest of scanlines with a pre-chosen, given number of iterations. Therefore, we propose to design the CCF-ILC filters $Q(j \omega)$ and $L(j \omega)$ for repetitively imaging on one scanline as in [6], and then quantify the size of the allowable line-to-line sample variation such that the imaging on the rest of scanlines can be proceeded with no iterations (i.e., scan once at each scanline).

Remark 1: The proposed CCF-ILC approach aims at achieving high-speed imaging of samples with relatively smooth topography (i.e., the line-to-line sample variation is relatively small).

\section{B. Controllers Design}

As in [6], a two-step approach to design the CCF-ILC filters is proposed and, in the following, we call $L(j \omega)$ the 'inversion-based ILC filter' and $Q(j \omega)$ the 'roll-off ILC filter'.

Design of the inversion-based ILC filter $L(j \omega)$

The inversion-based ILC filter $L(j \omega)$ is designed to minimize the term $\left|1-G_{P D}(j \omega) S(j \omega) L(j \omega)\right|$ in the iteration coefficient $\rho(\omega)$, by using the recently developed robust inversion technique [10]. We start with defining the model uncertainties as

$$
\Delta G(j \omega)=\frac{G_{L}(j \omega)}{G_{L, m}(j \omega)}=\Delta r(\omega) \cdot e^{j \Delta \theta(\omega)},
$$


where $G_{L}(j \omega)$ denotes the true linear dynamics response of the system, e.g., for the $z$-axis AFM dynamics,

$$
G_{L}(j \omega)=G_{P D}(j \omega) S(j \omega),
$$

and $G_{L, m}(j \omega)$ denotes the model of the linear dynamics $G_{L}(j \omega)$. Then the inversion-based ILC filter $L(j \omega)$ is designed as

$$
L(j \omega)=\alpha_{o p t}(\omega) \cdot G_{L, m}^{-1}(j \omega),
$$

where $\alpha_{\text {opt }}(\omega)$ is the optimal frequency-dependent gain to compensate for the dynamics uncertainty of the system $G_{L}(j \omega)$, i.e., the optimal gain $\alpha_{\text {opt }}(j \omega)$ is sought to minimize the feedforward tracking error, $\left|1-G_{P D}(j \omega) S(j \omega) L(j \omega)\right|$, with the presence of the dynamics uncertainty $\Delta G(j \omega)$,

$$
\begin{gathered}
\min _{L(j \omega)} \sup _{\Delta G}\left|1-G_{P D}(j \omega) S(j \omega) L(j \omega)\right|= \\
\min _{\alpha(j \omega)} \sup _{\Delta G}|1-\alpha(\omega) \Delta G(j \omega)| .
\end{gathered}
$$

It can be shown that the optimal gain $\alpha_{o p t}(j \omega)$ is given as [10]

$$
\alpha_{\text {opt }}(\omega)= \begin{cases}\frac{2 \cos \left(\Delta \theta_{m}(\omega)\right)}{\Delta r_{\min }(\omega)+\Delta r_{\max }(\omega)} & \omega \in \Omega_{c}, \\ 0 & \text { otherwise. }\end{cases}
$$

where $\Delta r_{\min }, \Delta r_{\max }$ and $\Delta \theta_{m}$ are the bounds to quantify the system uncertainties, i.e.,

$$
\begin{aligned}
\Delta r(j \omega) & \in\left[\Delta r_{\min }(j \omega), \Delta r_{\max }(j \omega)\right] \\
|\Delta \theta(j \omega)| & \leq\left|\Delta \theta_{m}(j \omega)\right|<\pi / 2 \quad \text { for } \omega \in \Omega_{c}
\end{aligned}
$$

and the set $\Omega_{c}$ contains the frequencies where the phase variation is less than $\pi / 2$, i.e.,

$$
\Omega_{c} \triangleq\left\{\omega \in \Omega_{c}|| \Delta \theta(j \omega) \mid<\pi / 2 \text { for } \omega \in \Omega_{c}\right\} .
$$

Then with the robust inverse filter $(13,15)$, the term $\left|1-G_{P D}(j \omega) S(j \omega) L(j \omega)\right|$ is minimized for bounded dynamics uncertainties,

$$
\begin{aligned}
& \min _{\alpha} \sup _{\Delta G}\left|1-G_{P D}(j \omega) S(j \omega) L(j \omega)\right| \\
& = \begin{cases}\frac{\cos \left(\Delta \theta_{m}(\omega)\right) \sqrt{2 \Delta r_{\min }^{2}(\omega)+\Delta r_{\max }^{2}(\omega)}}{\Delta r_{\min }(\omega)+\Delta r_{\max }(\omega)}<1 & \omega \in \Omega_{c}, \\
1 & \text { otherwise. }\end{cases}
\end{aligned}
$$

\section{Design of the roll-off ILC filter $Q(j \omega)$}

Secondly, we design the roll-off ILC filter $Q(j \omega)$ as a zerophase low-pass filter to ensure the convergence of the $\mathrm{CCF}$ ILC algorithm, and render a small ultimate error $e_{\infty}(j \omega)$ (see Eq. (4)). To facilitate the implementation, the zero-phase low-pass filter $Q(j \omega)$ is given in $z$-transfer function in the discrete-time:

$$
Q(z)=b_{0}+\sum_{k=1}^{N}\left(b_{k} z^{k}\right)+\sum_{k=1}^{N}\left(b_{k} z^{-k}\right)
$$

where the coefficients $b_{k} \in \Re$. It can be easily verified that the frequency response of $Q\left(e^{j \omega T}\right)$ is real-thus the phase of $Q(z)$ is zero. The ILC roll-off filter $Q(j \omega)$ is realized by combining a linear phase FIR low-pass filter with a linear phase lead as discussed in [6] and the bandwidth of the ILC roll-off filter $Q(j \omega)$ can be tuned during the experiments to assure the convergence.

\section{TRACKING IMPROVEMENT}

To show that the CCF-ILC approach can enhance the total imaging performance over feedback control alone, the tracking error of using the CCF-ILC approach is compared with that of using the feedback control alone (the second design objective). We first discuss the enhancement when the line-to-line sample variation is ignored (i.e., when using the CCF-ILC algorithm to repetitively image on the same scanline).

\section{A. No line-to-line sample variation case}

It can be shown that in this case the ultimate error of the CCF-ILC approach, $\left|e_{\infty}(\cdot)\right|$, is less than or equal to the residual error with feedback only, $\left|e_{F B}(\cdot)\right|=|S(j \omega) d(j \omega)|$, when the noise is negligible, i.e., $\delta(\omega)=0$. By Eq. (4), when the noise is ignored, the ratio of the ultimate error of the CCF-ILC approach with respect to the feedback control error, $q(\omega)$, becomes

$$
\begin{aligned}
q(\omega) & =\frac{\left|e_{\infty}(j \omega)\right|}{\left|e_{F B}(j \omega)\right|}=\frac{|1-Q(j \omega)|}{|1-\rho(\omega)|} . \\
& =\frac{|1-Q(j \omega)|}{|1-| Q(j \omega)|| 1-G_{P D}(j \omega) S(j \omega) L(j \omega) \mid}{ }^{19)}
\end{aligned}
$$

Then the discussion is proceeded by considering the tracking within and outside the frequency set $\Omega_{c}$ separately,

1) In the frequency set $\Omega_{c}$ where the dynamics variation is small and the CCF-ILC controller $L(j \omega)$ is applied, the term $\left|1-G_{P D}(j \omega) S(j \omega) L(j \omega)\right|$ in the error ratio (19) is less than one (as shown in (17)). Consequently, the error ratio is less than one, $q(\omega)<1$, because the ILC filter $Q(j \omega)$ is a zero phase low-pass filter and $Q(j \omega) \leq 1$ for frequency $\omega \in \Omega_{c} ;$

2) At other frequencies where the dynamics variation is large and the CCF-ILC controller $L(j \omega)$ is set to zero, the term $\left|1-G_{P D}(j \omega) S(j \omega) L(j \omega)\right|=1$ (see (17)), and thereby the error ratio $q(\omega)$ equals to one.

Thus by the continuity of the system dynamics, the above discussion implies that when the noise/disturbance is small and the line-to-line variation is ignored, the use of the proposed CCF-ILC approach will enhance the tracking performance.

\section{B. Line-to-line variation case}

Next, we consider the more general case where the line-toline sample profile variation occurs at each iteration, i.e., $\left|d_{k}-d_{k+1}\right| \geq 0$ for $k \geq 1$. Similar in Section III-A, the CCF-ILC ultimate error, $e_{\infty}(j \omega)$, is compared with the error of feedback only, $\left|e_{F B, \max }(\cdot)\right| \triangleq|S(j \omega)|\left|d_{\max }\right|$, where $d_{\max }=\max _{k} d_{k}$ (Note the ultimate error $e_{\infty}(j \omega)$ is the upper bound of the limit of the residual error when using the CCF-ILC). The following lemma provides a sufficient condition to guarantee that the CCF-ILC approach will improve the tracking over feedback alone.

Lemma 2: Let the noise effect $n_{k}$ be negligible, the use of the proposed CCF-ILC approach will enhance the tracking performance, i.e.

$$
\left|e_{\infty}(j \omega)\right| \leq\left|e_{F B, \max }(j \omega)\right| \text { for } \omega \in \Omega_{c},
$$


if the relative line-to-line sample variation $\Delta R_{d}(\%)$ is bounded as

$$
\Delta R_{d}(\%) \triangleq\left|\frac{\widehat{\Delta d}_{\max }(j \omega)}{d_{\max }(j \omega)}\right| \leq|1-\rho(\omega)| \times 100 \%
$$

Lemma 2 can be easily proved by using Eq. (4) in Lemma 1. Note that in the low frequency range, the dynamics uncertainty tends to be small, so are the noise/disturbance effects. As a result, the inversion-based ILC filter $L(j \omega)$ can be chosen to render the iterative coefficient $\rho \rightarrow 0$, and the rolloff filter $Q(j \omega)$ can be chosen to be one. Therefore, the above Eq. (21) implies that as large as nearly $100 \%$ line-to-line sample variation can be allowed in the low-frequency range (or alternatively, when the imaging speed is relatively low). Next, we introduce a more conservative sufficient condition which quantifies the allowable line-to-line sample variation in terms of the line-to-line variation directly (instead of the modified line-to-line variation $\widehat{\Delta d}_{\max }(j \omega)$, which depends on the ILC filter $Q(j \omega)$ ).

\section{EXPERIMENTAL EXAMPLE}

We implement the proposed CCF-ILC approach on an AFM system (Dimension ${ }^{T M}$ 3100, Veeco Inc.). The efficacy of the CCF-ILC approach to improve the imaging on one scanline (over using feedback control only) has been demonstrated through experiments in [6]. In this paper, we focus on the use of CCF-ILC method to obtain an entire image. Note that the CCF-ILC law was designed by following the procedure described in Sec. II-B, similar to the experiment example described in [6].

The proposed CCF-ILC technique was implemented to scan a calibration sample (TGZ02, from MikroMasch) where the nominal pitch size is $3 \mu \mathrm{m}$, and the nominal step height is $84 \mathrm{~nm}$. The image area is $20 \mu \mathrm{m} \times 20 \mu \mathrm{m}$, and the precision positioning in the lateral $x-y$ scanning was achieved by using the inversion-based iterative control (IIC) approach [3]. In this experiments, less than $1 \%$ of the maximum relative tracking error in the lateral scanning was achieved by using the IIC technique for the three different scan rates, $16 \mathrm{~Hz}$, $32 \mathrm{~Hz}$, and $64 \mathrm{~Hz}$ (The imaging results of lower scan rate at $8 \mathrm{~Hz}$ was not shown).

The experimental results show that the imaging speed can be significantly improved by using the proposed CCF-ILC approach - much smaller residual error was obtained than that by using the feedback control only. At the scan rate of $16 \mathrm{~Hz}$, the maximum residual error under the CCF-ILC approach was 3 times smaller than that under the PI control, as shown in Fig. 2 (a1) (b1). Such large reduction of the residual error by using the proposed CCF-ILC technique over the PI control was maintained even when the equivalent scan rate was doubled $(32 \mathrm{~Hz})$ and tripled $(64 \mathrm{~Hz})$ as shown in Fig. 2 (a2), (b2), (a3) and (b3). Therefore, the experimental results demonstrated the efficacy of the proposed CCF-ILC approach in improving the AFM imaging speed.

The experimental results also showed that the sample estimation at high-speed can also be significantly improved with the use of the CCF-ILC approach, even when there existed large cross-axis dynamics-coupling effect. The sample estimation
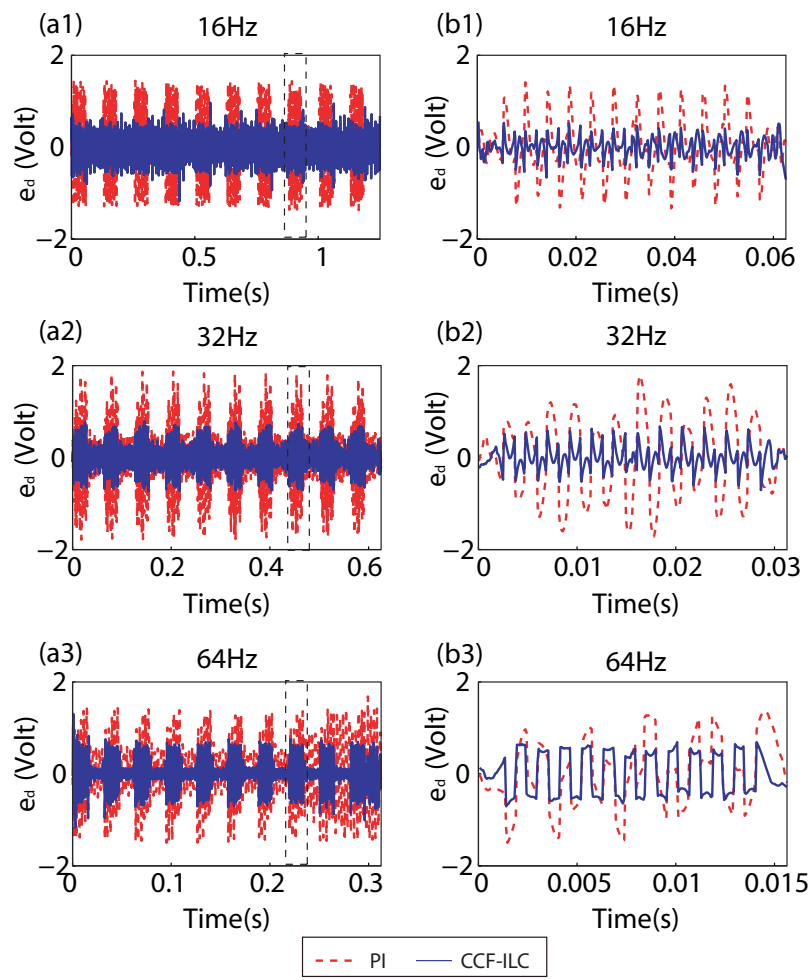

Fig. 2. Comparison of the deflection error (the residual error) by using the proposed CCF-ILC approach with that by using the PI control for four different equivalent scan rates of (a1, b1) $8 \mathrm{~Hz},(\mathrm{a} 2, \mathrm{~b} 2) 16 \mathrm{~Hz},(\mathrm{a} 3, \mathrm{~b} 3)$ $32 \mathrm{~Hz}$, and $(\mathrm{a} 4, \mathrm{~b} 4) 64 \mathrm{~Hz}$ in sample imaging, where the left column shows the total of 10 repeated scan result; and the right column is the zoomed-in view of the one scanline result in the dashed window in the left column. The effective lateral scan range is $20 \mu \mathrm{m}$.

results obtained by using the CCF-ILC approach and those by using the PI control approach are compared in Fig. 3 for the three different scan rates. Note that the dynamics coupling effect were removed from the sample estimation for the CCF-ILC approach as in [6]. At the scan rate of $16 \mathrm{~Hz}$, the estimated sample profile obtained by using the CCF-ILC approach was close to the true sample profile (the rectangle pitches can be clearly identified), whereas the estimated sample profile by using the PI control showed pronounced sample distortions (the top of the pitches were cornered around instead of being flat, see Fig. 3 (a)). As the scan rate increased (to $32 \mathrm{~Hz}$ ), the estimation error became larger by using the PI control (where not only the top but also the bottom of the pitches were cornered around), while the sample profile can still be relatively well estimated by using the proposed approach. When the scan rate was increased to $64 \mathrm{~Hz}$, the imaging distortion was even more pronounced under the PI control - the basic shape of the sample surface can not be recognized, and large variations occurred among the estimated pitches. On the contrary, by using the CCF-ILC approach, the sample estimation signal was still relatively close to the sample profile. Therefore, the experiment results showed that the proposed CCF-ILC approach can achieve a smaller tracking error as well as a better sample estimation than those obtained by using the feedback control alone. 

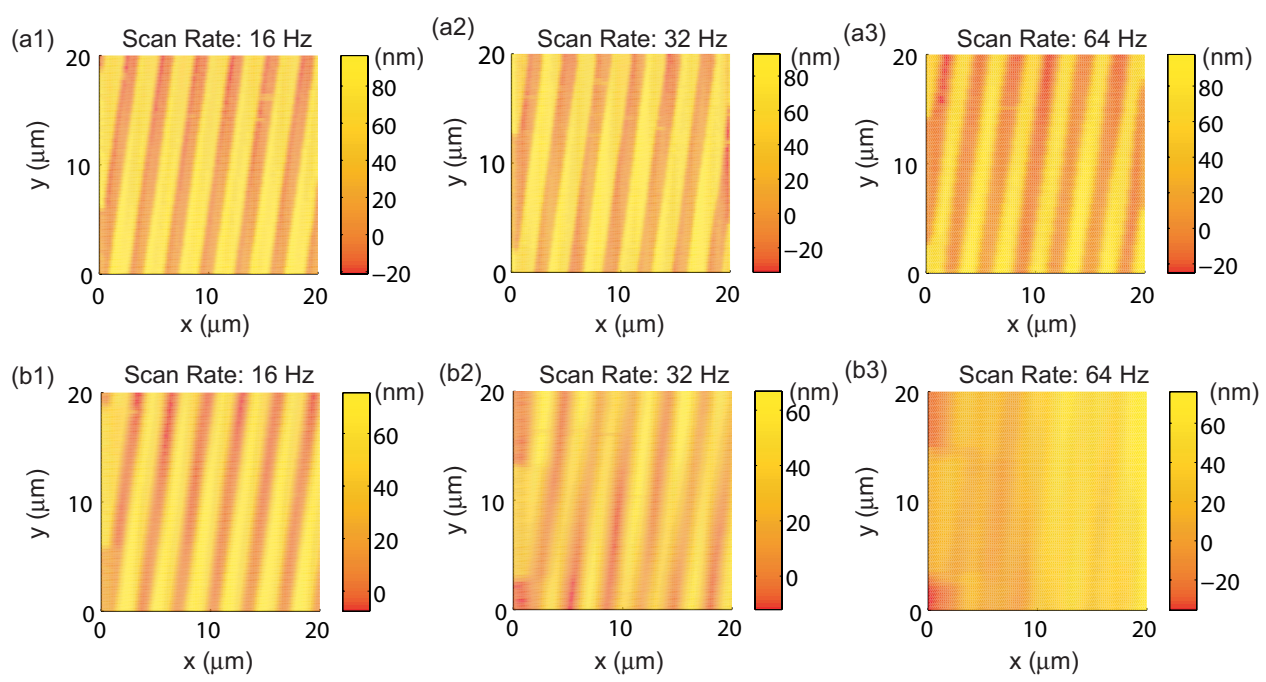

Fig. 3. Comparison of the sample estimation results by using (the upper row) the proposed CCF-ILC approach with that by using (the bottom row) the PI control for three different equivalent scan rates of $(\mathrm{a} 1, \mathrm{~b} 1) 16 \mathrm{~Hz},(\mathrm{a} 2, \mathrm{~b} 2) 32 \mathrm{~Hz}$, and $(\mathrm{a} 3, \mathrm{~b} 3) 64 \mathrm{~Hz}$. The lateral scan range is $20 \mu \mathrm{m}$.
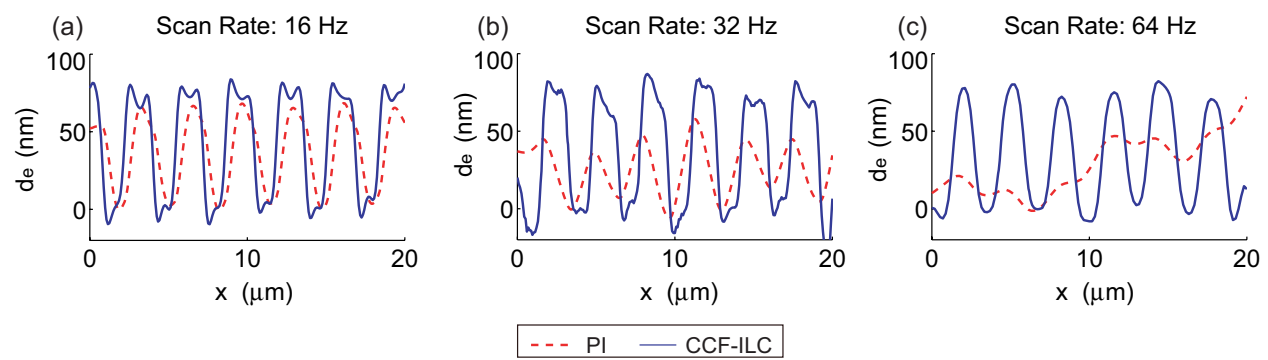

Fig. 4. Comparison of the sample estimation results (cross section view) by using the proposed CCF-ILC approach with that by using the PI control for three different equivalent scan rates of (a) $16 \mathrm{~Hz}$, (b) $32 \mathrm{~Hz}$, and (c) $64 \mathrm{~Hz}$. The lateral scan range is $20 \mu \mathrm{m}$.

\section{CONCLUSION}

The CCF-ILC approach was extended in this article from high-speed AFM imaging of one scanline to the entire imaging of the samples. First, the convergence (stability) of the CCF-ILC law was analyzed for the general case where the line-to-line sample profile variation (i.e., the desired trajectory in general) occurred at each iteration. The conditions for the convergence of the CCF-ILC approach were characterized. Secondly, the allowable line-to-line sample variation was quantified for the CCF-ILC approach so that the imaging on the rest of the scanlines can be proceeded with no iterations. Then the proposed CCF-ILC control approach was illustrated by implementing it to the $z$-axis direction control in AFM imaging. Experimental results show that the imaging speed as well as the sample estimation can be significantly improved by using the proposed approach.

\section{ACKNOWLEDGMENT}

The financial support from NSF Grants CMMI-0626417 and DUE-0632908 is gratefully acknowledged.

\section{REFERENCES}

[1] G. Binnig, C. F. Quate, and C. Gerber, "Atomic force microscope," Physical Review Letters, vol. 56, no. 9, pp. 930-934, 1986.
[2] K.-B. Lee, S.-J. Park, C. A. Mirkin, J. C. Smith, and M. Mrksich, "Protein nanoarrays generated by dip-pen nanotithography," Science, vol. 295, no. 5560, pp. 1702-1705, 2002.

[3] Y. Wu and Q. Zou, "Iterative control approach to compensate for both the hysteresis and the dynamics effects of piezo actuators," IEEE Trans. on Control Systems Technology, vol. 15, pp. 936-944, 2007.

[4] S. Salapaka, A. Sebastian, J. P. Cleveland, and M. V. Salapaka, "High bandwidth nano-positioner: A robust control approach," Review of Scientific Instruments, vol. 73(9), pp. 3232-3241, Sept., 2002.

[5] G. Schitter, A. Stemmer, and F. Allgower, "Robust 2DOF-control of a piezoelectric tube scanner for high-speed atomic force microscopy,"

[6] Y. Wu, Q. Zou, and C. Su, "A current cycle feedback iterative learning control approach to AFM imaging," in Proceedings of American Control Conference, (Seattle), IEEE, June 2008.

[7] S. Skogestad and I. Postlethwaite., Multivariable Feedback control: Analysis and Design. New York: Wiley, second ed., 2005.

[8] S. Devasia, D. Chen, and B. Paden, "Nonlinear inversion-based output tracking," IEEE Trans. on Automatic Control, vol. 41, no. 7, pp. 930942, 1996.

[9] Q. Zou and S. Devasia, "Preview-based optimal inversion for output tracking: Application to scanning tunneling microscopy," IEEE Trans. on Control Systems Technology, vol. 12, pp. 375-386, May 2004.

[10] Y. Wu and Q. Zou, "Robust-inversion-based 2DOF-control design for output tracking: Piezoelectric actuator example," in IEEE conference on Decision and Control, (New Orleans, LA), pp. 2451-2457, Dec., 20072007. 